\title{
Investigation of Two Qubit Quantum Gates in Linear Optics
}

\author{
T.C.Ralph, N.K.Langford, T.B.Bell, J.L.O’Brien, G.J.Pryde, A.G.White and G.J.Milburn \\ Centre for Quantum Computer Technology, Department of Physics \\ University of Queensland, QLD 4072, Australia
}

\begin{abstract}
We discuss progress towards implementing two qubit quantum gates in optics. We review the operation of an optical quantum gate which performs all the operations of a control-NOT (CNOT) gate in the coincidence basis with two, unentangled photons as the input and discuss its implementation.
\end{abstract}

Keywords: Quantum Computation, Quantum Optics, Quantum Information

\section{INTRODUCTION}

Quantum information processing requires qubits which are strongly isolated from the general enviroment and yet interact strongly with controlled environments such that they can be manipulated and measured accurately. Qubits based on the polarization state of individual photons have the advantage of low decoherence rates in free propagation and can be accurately manipulated and measured at the single qubit level. Optical down conversion experiments have been very successful in producing and analyzing a large range of two photon entangled states. ${ }^{1-3}$

The major source of decoherence in such systems is photon loss. However, because this results in the total destruction of the qubit it can easily be screened for by only excepting results in which all qubits arrive at the measurement station. This is referred to as working in the coincidence basis such that only events where two photons are detected in the same, narrow time window are recorded. The entangled state postselected this way may be a pure Bell state even though the total state is non-deterministic and may have experienced considerable mixing from photon loss. This "screening" procedure results in an exponentially dropping signal with the size of the circuit and so such systems are not scalable in the quantum computational sense in their present form. None-the-less they provide an excellent testing ground for quantum information concepts. Useful application of this type of technology seems much closer in the realm of quantum communications.

A key two qubit gate is the Controlled Not (CNOT) gate. A deterministic CNOT gate would require either very high non-linearities ${ }^{4}$ or complex linear networks with detection and feedforward. ${ }^{5}$ Building on the latter ideas a number of linear, coincidence basis CNOTs have been described with quite different properties.

Sanaka $e t a l^{6}$ have demonstrated a gate which converts entanglement in one degree of freedom; time-energy, into CNOT gate operation on another degree of freedom; polarization. An entangled state fringe visibility of 0.44 was observed in this experiment. Dür ${ }^{7}$ and Briegel have shown that in the presence of efficient, local quantum non-demolition measurements such a techniques could be made scalable. However no practical means is yet known for doing this in optics.

More recently Pittman et al ${ }^{8}$ have described a 3 -photon CNOT gate. This gate is related to the teleportation gate described by Knill, Laflamme and Milburn (KLM) ${ }^{5}$ which uses entangled ancilla photons and detection to implement gate operation. In the experiment a single, unentangled ancilla photon was used instead of entangled ancilla, but the trade-off was the need to work in the coincidence basis. An entangled state fringe visibility of 0.61 was observed.

In this paper we discuss a linear, coincidence basis gate which performs all the operations of a CNOT gate and requires only an unentangled two photon input. 9,10 This gate is closely related to the fundamental

Further author information: (Send correspondence to TCR)

TCR: E-mail: ralph@physics.uq.edu.au 

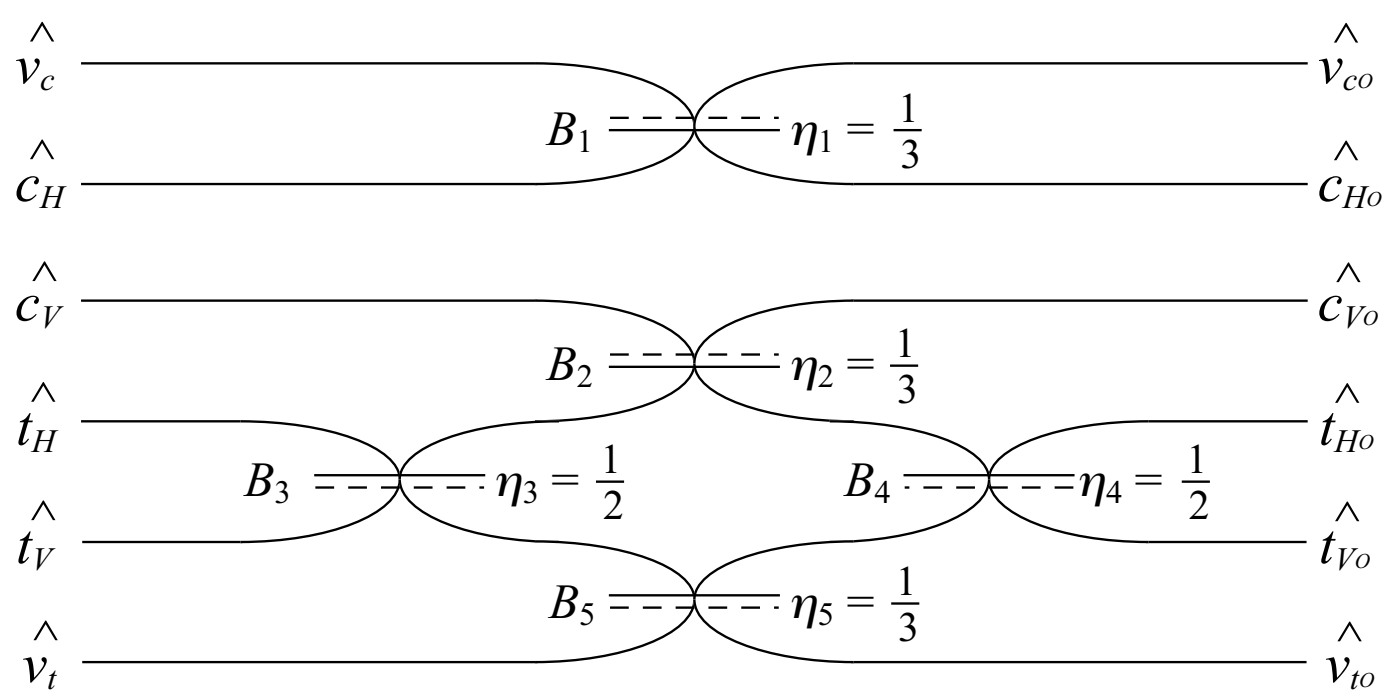

Figure 1. Schematic of the gate. Dashing indicates the surface from which a sign change occurs upon reflection. The control modes are $c_{H}$ and $c_{V}$. the target modes are $t_{H}$ and $t_{V}$. the modes $v_{c}$ and $v_{t}$ are unoccupied ancillary modes.

gates described by KLM ${ }^{5}$ which are envisaged as producing the entanglement for the teleportation gates. The simplicity and versatility of this gate suggests that in addition to being a testing ground for the ideas of KLM it could also be a good candidate for in principle demonstrations of small scale quantum circuits and possible applications in quantum communications. We expect to report an experimental demonstration of this gate in the near future.

The paper is arranged in the following way. In section 2 we describe the gates construction and operation conceptually. In section 3 we consider the effect of imperfections in its construction, particularly focusing on the effect of beamsplitter and mode-matching errors on the gates efficacy as a Bell state analyzer. In section 4 we briefly discuss a possible experimental arrangement and then conclude in section 5 .

\section{THE GATE}

In this section we describe the two photon CNOT gate under ideal conditions. ${ }^{9}$ The gate is shown in Fig.1. All beamsplitters, $B 1, B 2, B 3, B 4$, and $B 5$, are assumed asymmetric in phase. That is, it is assumed that the operator input/output relations (the Heisenberg equations) between the two input mode operators ( $a_{i n}$ and $\left.b_{i n}\right)$ and the corresponding output operators $\left(a_{\text {out }}\right.$ and $\left.b_{\text {out }}\right)$ for the beamsplitters have the general form

$$
\begin{aligned}
& a_{\text {out }}=\sqrt{\eta} a_{i n}+\sqrt{1-\eta} b_{i n} \\
& b_{\text {out }}=\sqrt{1-\eta} a_{i n}-\sqrt{\eta} b_{i n}
\end{aligned}
$$

where $\eta(1-\eta)$ is the reflectivity (transmittivity) of the beamsplitter. Reflection off the bottom produces the sign change except for $B 1$ and $B 2$ which have a sign change by reflection off the top. This phase convention simplifies the algebra but other phase relationships will work equally well in practice. Beamsplitters $B 3$ and $B 4$ are both 50:50 $(\eta=1 / 2)$. The beamsplitters $B 1, B 2$ and $B 5$ have equal reflectivities of one third $(\eta=1 / 3)$.

We employ dual rail logic such that the "control in" qubit is represented by the two bosonic mode operators $c_{H}$ and $c_{V}$. A single photon occupation of $c_{H}$ with $c_{V}$ in a vacuum state will be our logical 0 , which we will write $|H\rangle_{c}$ (to avoid confusion with the vacuum state). Whilst a single photon occupation of $c_{V}$ with $c_{H}$ in a vacuum state will be our logical 1, which we will write $|V\rangle_{c}$. Superposition states can also be formed via beamsplitter interactions. Similarly the "target in" is represented by the bosonic mode operators $t_{H}$ and $t_{V}$ and the states $|H\rangle_{t}$ and $|V\rangle_{t}$, with the same interpretations as for the control. The use of $H$ and $V$ to describe the 
states of the qubits of course alludes to the usual encoding in polarization. To go from polarization encoding to dual rail spatial encoding and vice versa in the lab requires a polarizing beamsplitter and half-wave plate. An implementation based specifically on polarization encoding is discussed in section 4.

The Heisenberg equations relating the control $\left(c_{H}, c_{V}\right)$ and target $\left(t_{H}, t_{V}\right)$ input modes to the their corresponding outputs are

$$
\begin{aligned}
c_{H_{O}} & =\frac{1}{\sqrt{3}}\left(\sqrt{2} v_{c}+c_{H}\right) \\
c_{V_{O}} & =\frac{1}{\sqrt{3}}\left(-c_{V}+t_{H}+t_{V}\right) \\
t_{H_{O}} & =\frac{1}{\sqrt{3}}\left(c_{V}+t_{H}+v_{t}\right) \\
t_{V_{O}} & =\frac{1}{\sqrt{3}}\left(c_{V}+t_{V}-v_{t}\right) \\
v_{c_{O}} & =\frac{1}{\sqrt{3}}\left(-v_{c}+\sqrt{2} c_{H}\right) \\
v_{t_{O}} & =\frac{1}{\sqrt{3}}\left(t_{H}+t_{V}-v_{t}\right)
\end{aligned}
$$

Ancillary, vacuum input modes, $v_{c}$ and $v_{t}$, complete the network. The gate operates by causing a sign shift in the interferometer formed by the splitting and remixing of the target modes, conditional on the presence of a photon in the $c_{V}$ mode. Thus the target modes swap if the control is in the state $|V\rangle_{c}$ but do not if the control is in state $|H\rangle_{c}$. This is always true when a coincidence is measured between the control and target outputs (photons are detected at the same time). However such coincidences only occur one ninth of the time, on average. The other eight times out of nine either the target or the control or both do not contain a photon, that is one or both of the qubits are destroyed. This can be seen explicitly by calculating the output state of the system in the Schrödinger picture. Consider the general input state

$$
\begin{aligned}
|\phi\rangle & =(\alpha|H H\rangle+\beta|H V\rangle+\gamma|V H\rangle+\delta|V V\rangle)|00\rangle \\
& =\left(\alpha c_{H}^{t} t_{H}+\beta c_{H}^{t} t_{V}+\gamma c_{V}^{t} t_{H}+\delta c_{V} t_{V}\right)|0000\rangle|00\rangle
\end{aligned}
$$

where the ordering in the kets is $\left|n_{c H} n_{c V} n_{t H} n_{t V}\right\rangle\left|n_{v c} n_{v t}\right\rangle$ with $n_{c H}=c_{H}^{\dagger} c_{H}$ etc and we use the short hand $|1010\rangle=|H H\rangle$ etc where appropriate. For a time symmetric linear network such as that in Fig.1, the output state can be directly obtained from the input state, Eq.3, by substituting input operators for the output operators given by Eq.2. ${ }^{11}$ Thus we obtain

$$
\begin{aligned}
|\phi\rangle_{\text {out }}= & \left(\alpha c_{H_{O}}^{\dagger} t_{H_{O}}^{\dagger}+\beta c_{H_{O}}^{\dagger} t_{V_{O}}^{\dagger}+\gamma c_{V_{O}}^{\dagger} t_{H_{O}}^{\dagger}+\delta c_{V_{O}}^{\dagger} t_{V_{O}}^{\dagger}\right)|0000\rangle|00\rangle \\
= & \frac{1}{3}\{\alpha|H H\rangle+\beta|H V\rangle+\gamma|V V\rangle+\delta|V H\rangle \\
& +\sqrt{2}(\alpha+\beta)|0100\rangle|10\rangle+\sqrt{2}(\alpha-\beta)|0000\rangle|11\rangle+(\alpha+\beta)|1100\rangle|00\rangle \\
& +(\alpha-\beta)|1000\rangle|01\rangle+\alpha|0010\rangle|10\rangle+\beta|0001\rangle|10\rangle \\
& -(\gamma+\delta)|0200\rangle|00\rangle-(\gamma-\delta)|0100\rangle|01\rangle+\gamma|0020\rangle|00\rangle \\
& +(\gamma-\delta)|0010\rangle|01\rangle+(\gamma+\delta)|0011\rangle|00\rangle+(\gamma-\delta)|0001\rangle|01\rangle+\delta|0002\rangle|00\rangle\}
\end{aligned}
$$

The state postselected in the coincidence basis is then just

$$
|\phi\rangle_{c b}=\alpha|H H\rangle+\beta|H V\rangle+\gamma|V V\rangle+\delta|V H\rangle
$$

occurring with probability one ninth. The relationship between Eq.3 and Eq.5 is a CNOT transformation.

It is also useful to look at the coincidence number expectation values, obtained directly from the Heisenberg equations (Eq.2). These can be interpreted as the predicted output coincident count rates normalized to the 


\begin{tabular}{|c|c|c|c|c|}
\hline Input & $\left\langle n_{c H_{O}} n_{t H_{O}}\right\rangle$ & $\left\langle n_{c H_{O}} n_{t V_{O}}\right\rangle$ & $\left\langle n_{c V_{O}} n_{t H_{O}}\right\rangle$ & $\left\langle n_{c V_{O}} n_{t V_{O}}\right\rangle$ \\
\hline \hline$|H\rangle_{c}|H\rangle_{t}$ & $\frac{1}{9}$ & 0 & 0 & 0 \\
\hline$|H\rangle_{c}|V\rangle_{t}$ & 0 & $\frac{1}{9}$ & 0 & 0 \\
\hline$|V\rangle_{c}|H\rangle_{t}$ & 0 & 0 & 0 & $\frac{1}{9}$ \\
\hline$|V\rangle_{c}|V\rangle_{t}$ & 0 & 0 & $\frac{1}{9}$ & 0 \\
\hline
\end{tabular}

Table 1. Coincident expectation values calculated for each of the four logical basis inputs.

input pair rate. An example is given in Table 1 which shows the count rates for logical basis inputs. A more interesting case is to use the four Bell-states,

$$
\begin{aligned}
\left|\psi^{ \pm}\right\rangle & =\frac{1}{\sqrt{2}}\left(|H\rangle_{c}|H\rangle_{t} \pm|V\rangle_{c}|V\rangle_{t}\right) \\
\left|\phi^{ \pm}\right\rangle & =\frac{1}{\sqrt{2}}\left(|H\rangle_{c}|V\rangle_{t} \pm|V\rangle_{c}|H\rangle_{t}\right)
\end{aligned}
$$

as inputs and to detect the control in the superposition basis by mixing the control outputs on a 50:50 beamsplitter before detection:

$$
\begin{aligned}
c_{S_{1}} & =\frac{1}{\sqrt{2}}\left(c_{H_{O}}+c_{V_{O}}\right) \\
c_{S_{2}} & =\frac{1}{\sqrt{2}}\left(c_{H_{O}}-c_{V_{O}}\right)
\end{aligned}
$$

In Table 2 the count rates for this arrangement are presented showing the ability to distinguish all four Bell states (albeit with non unit efficiency). Such a Bell state analyzer could have significant applications in quantum communications. In the next section we will use this application as an example in order to investigate the effect of non-optimal parameters on the gate.

\begin{tabular}{|c|c|c|c|c|}
\hline Input & $\left\langle n_{c S_{1}} n_{t H_{O}}\right\rangle$ & $\left\langle n_{c S_{2}} n_{t V_{O}}\right\rangle$ & $\left\langle n_{c S_{1}} n_{t H_{O}}\right\rangle$ & $\left\langle n_{c S_{2}} n_{t V_{O}}\right\rangle$ \\
\hline \hline$\left|\psi^{+}\right\rangle$ & $\frac{1}{9}$ & 0 & 0 & 0 \\
\hline$\left|\psi^{-}\right\rangle$ & 0 & $\frac{1}{9}$ & 0 & 0 \\
\hline$\left|\phi^{+}\right\rangle$ & 0 & 0 & $\frac{1}{9}$ & 0 \\
\hline$\left|\phi^{-}\right\rangle$ & 0 & 0 & 0 & $\frac{1}{9}$ \\
\hline
\end{tabular}

Table 2. Coincident expectation values calculated in the superposition basis for the four Bell states.

\section{NON-OPTIMAL OPERATION}

In this section we investigate the operation of the gate under non-optimal conditions. ${ }^{9}$ The accuracy with which the gate operates will be determined by how closely the parameters of the constructed gate correspond to those of the idealized gate of the previous section. We can identify three potential sources of error: incorrect beamsplitter ratios; non-unit mode matching and; timing errors. One advantage of working in the coincidence basis is that losses and detector inefficiency can be ignored because they take the system out of the coincidence basis and thus their only effect is to reduce the count rate.

Timing Errors. Correct gate operation depends on indistinguishability of the paths taken by the two photons through the network. This means that they must arrive simultaneously at the central beamsplitter to an accuracy of a fraction of their coherence length. Photon coherence length in down conversion experiments is generally determined by pre-detection frequency filtering and can be of order one hundred wave-lengths. Locking path lengths on this scale should not be a major problem.

Beamsplitter ratios. The effect of non-optimal beamsplitter ratios can be investigated by deriving the operator equations (Eq.2) more generally, with arbitrary beamsplitter ratios. For simplicity we assume that 

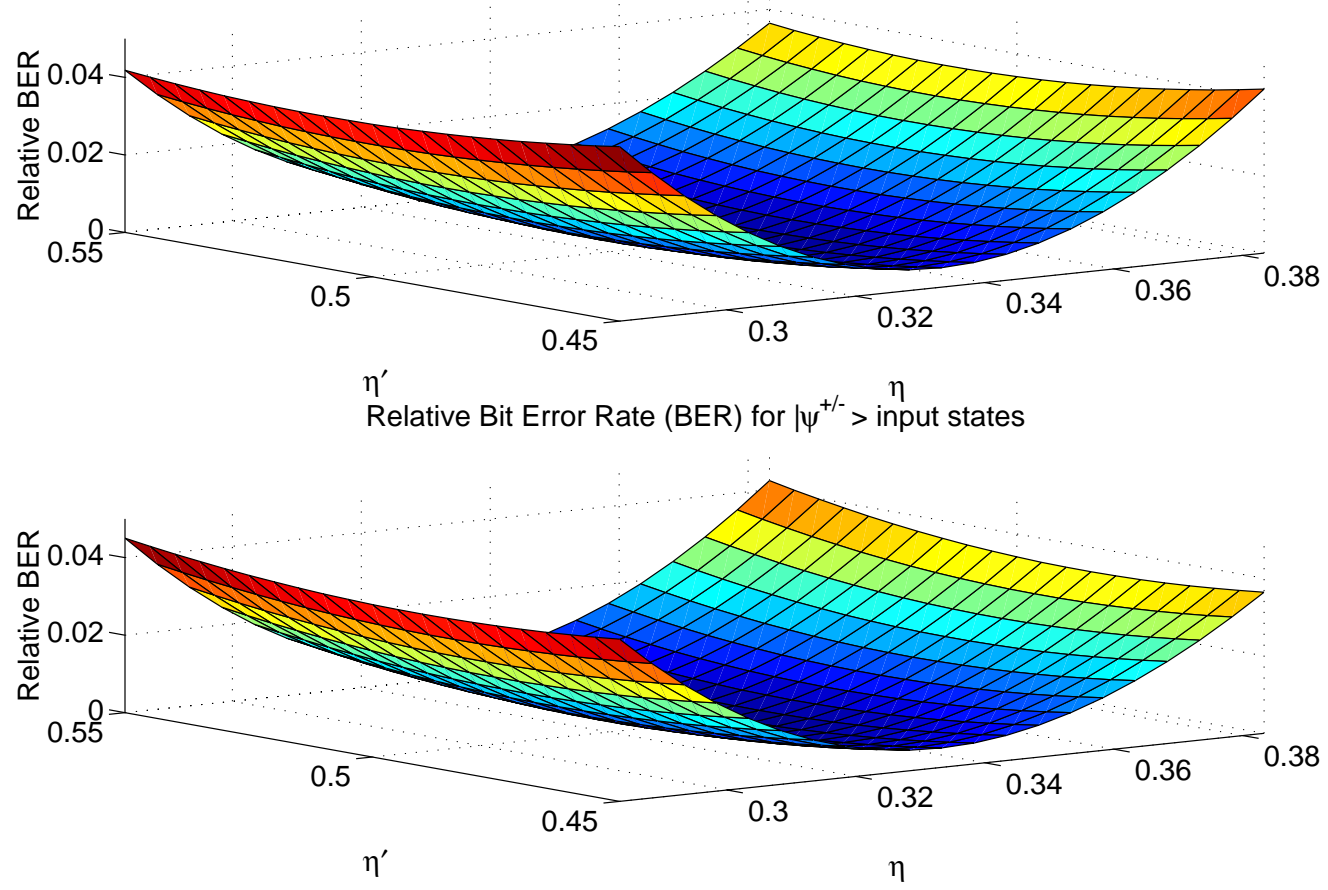

Figure 2. Relative error rates, i.e. error rate/total rate, for Bell state analysis as a function of beamsplitter ratios close to the optimum values of $\eta^{\prime}=1 / 2$ and $\eta=1 / 3$.

the beamspitters all came from the same "production-run" such that any deviation from the optimal value is common. That is, we might suppose that both the 50:50 beamsplitters actually have a reflectivity of $\eta^{\prime}$ whilst the three $1 / 3: 2 / 3$ beamsplitters all actually have reflectivities $\eta$. The Heisenberg equations are then

$$
\begin{aligned}
c_{H_{O}} & =\sqrt{\eta} c_{H}+\sqrt{1-\eta} v_{c} \\
c_{V_{O}} & =-\sqrt{\eta} c_{V}+\sqrt{(1-\eta) \eta^{\prime}} t_{H}+\sqrt{(1-\eta)\left(1-\eta^{\prime}\right)} t_{V} \\
t_{H_{O}} & =\sqrt{\eta}\left(1-2 \eta^{\prime}\right) t_{V}+2 \sqrt{\eta\left(1-\eta^{\prime}\right) \eta^{\prime}} t_{H}+\sqrt{\eta \eta^{\prime}} c_{V}+\sqrt{(1-\eta)\left(1-\eta^{\prime}\right)} v_{t} \\
t_{V_{O}} & =2 \sqrt{\eta\left(1-\eta^{\prime}\right) \eta^{\prime}} t_{V}+\sqrt{\eta}\left(1-2 \eta^{\prime}\right) t_{H}+\sqrt{(1-\eta)\left(1-\eta^{\prime}\right)} c_{V_{O}}-\sqrt{(1-\eta) \eta^{\prime}} v_{t} \\
v_{c_{O}} & =-\sqrt{\eta} v_{c}+\sqrt{1-\eta} c_{H_{O}} \\
v_{t_{O}} & =\sqrt{(1-\eta)\left(1-\eta^{\prime}\right)} t_{H}+\sqrt{(1-\eta) \eta^{\prime}} t_{V}-\sqrt{\eta} v_{t}
\end{aligned}
$$

In general the effect of varying the beamsplitter ratios is input state dependent. However for small deviations from the optimum values Bell state analysis is approximately state independent and serves as a useful diagnostic. In Fig. 2 we plot the error probability in distinguishing the Bell states as a function of $\eta$ and $\eta^{\prime}$ in the region close to their optimum values. The dependence of the error probability on $\eta^{\prime}$ is mirror imaged between the $\left|\psi^{ \pm}\right\rangle$and the $\left|\phi^{ \pm}\right\rangle$Bell states. However this dependence is negligible in the region close to $\eta^{\prime}=1 / 2$. The dependence on $\eta$ is more pronounced. For an $\eta$ of $1 / 3 \pm 0.01$ (and $\eta^{\prime}$ of $1 / 2 \pm 0.05$ ) error rates of about $0.7 \%$ are predicted. Such accuracy is achievable thus we conclude that errors below $1.0 \%$ should be realistic with current beamsplitter technology.

Mode matching errors. Mode matching in non-classical interference experiments is generally quite difficult and may be identified as a major contributor to non-unit visibility. Given the key role of non-classical interference in the CNOT gate we may expect mode matching errors to be of some significance. 


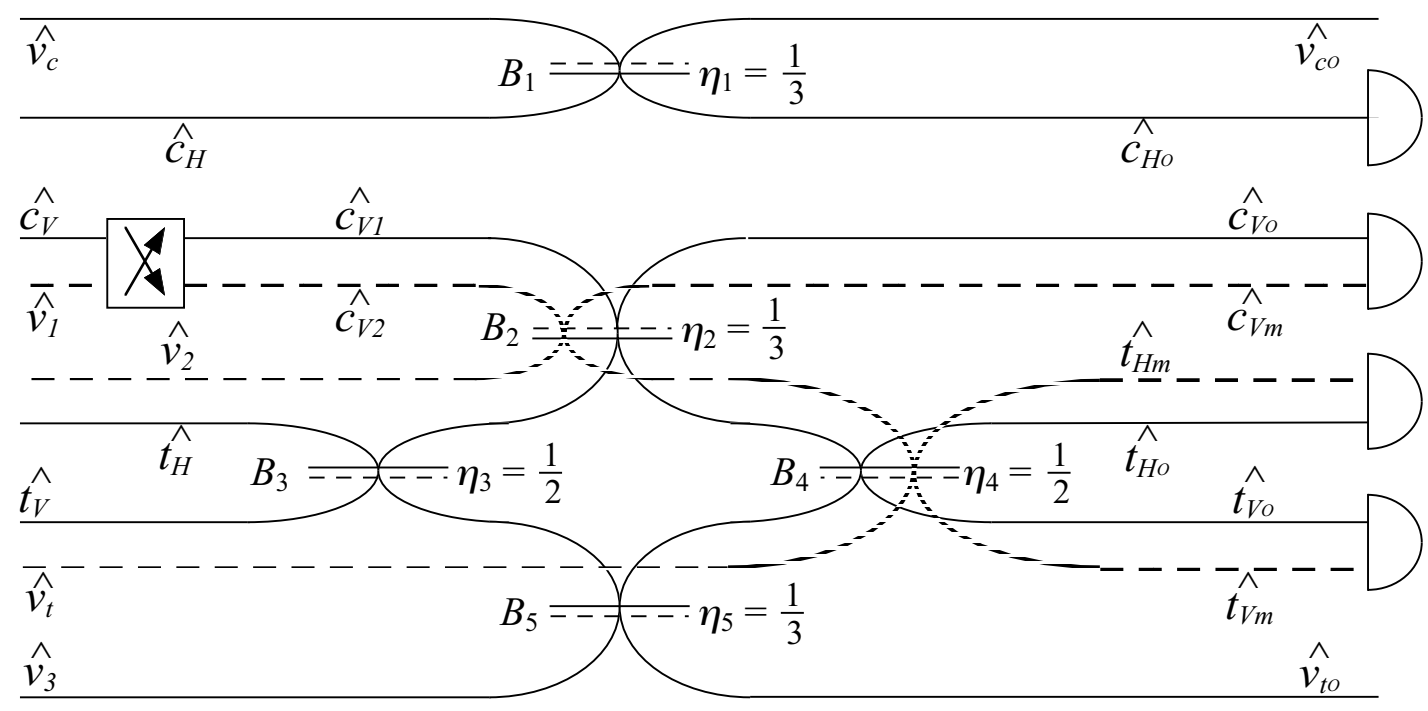

Figure 3. Schematic diagram of the coincidence CNOT gate including the effects of mode matching. The mismatch is represented by splitting $c_{V}$ into two orthogonal modes $c_{V 1}$ and $c_{V 2}$. Ancillary modes $v_{1}, v_{2}$ and $v_{3}$ interact with the propagating mismatch.

In order to model the mismatch of input modes at the central beamsplitter, ancillary modes $v_{1}, v_{2}$ and $v_{3}$ (originally in the vacuum state) are introduced to interact with the propagating mismatch mode. The additional output modes are labelled $c_{V_{m}}, c_{H_{m}}$ and $t_{V_{m}}$ (see Fig.3). The mode $c_{v}$ is assumed to be the source of the mismatch, after having passed through some kind of optical element that has misaligned it.

$$
\begin{aligned}
& c_{v_{1}}=\sqrt{\xi} c_{V}+\sqrt{1-\xi} v_{1} \\
& c_{v_{2}}=-\sqrt{1-\xi} c_{V}+\sqrt{\xi} v_{1}
\end{aligned}
$$

The parameter $\xi$ quantifies the degree of mode matching between the control and target modes at the central beamsplitter. So long as the modes are matched reasonably well, $c_{v_{1}}$ can be considered a sort of "primary" mode. It interacts with the output from beamsplitter $B_{3}$ in the same way as for the case neglecting mode matching. The mismatch component $c_{v_{2}}$ interacts only with the newly introduced vacuum modes.

The equations for the output modes of the quantum CNOT gate, including the effects of a mode mismatch, are

$$
\begin{aligned}
v_{c_{O}} & =\frac{1}{\sqrt{3}}\left(-v_{c}+\sqrt{2} c_{H}\right) \\
c_{H_{O}} & =\frac{1}{\sqrt{3}}\left(\sqrt{2} v_{c}+c_{H}\right) \\
c_{V_{O}} & =\frac{1}{\sqrt{3}}\left(-\sqrt{\xi} c_{V}-\sqrt{1-\xi} v_{1}+t_{H}+t_{V}\right) \\
c_{V_{m}} & =\frac{1}{\sqrt{3}}\left(\sqrt{1-\xi} c_{V}-\sqrt{\xi} v_{1}+\sqrt{2} v_{2}\right) \\
t_{H_{O}} & =\frac{1}{\sqrt{3}}\left(\sqrt{\xi} c_{V}+t_{H}+\sqrt{1-\xi} v_{1}+v_{t}\right) \\
t_{H_{m}} & =\frac{1}{\sqrt{3}}\left(-\sqrt{1-\xi} c_{V}+\sqrt{\xi} v_{1}+\frac{1}{\sqrt{2}} v_{2}+\sqrt{\frac{3}{2}} v_{3}\right) \\
t_{V_{O}} & =\frac{1}{\sqrt{3}}\left(\sqrt{\xi} c_{V}+t_{V}-\sqrt{1-\xi} v_{1}-v_{t}\right)
\end{aligned}
$$




$$
\begin{aligned}
t_{V_{m}} & =\frac{1}{\sqrt{3}}\left(-\sqrt{1-\xi} c_{V}+\sqrt{\xi} v_{1}+\frac{1}{\sqrt{2}} v_{2}-\sqrt{\frac{3}{2}} v_{3}\right) \\
v_{t_{O}} & =\frac{1}{\sqrt{3}}\left(t_{H}-t_{V}-v_{t}\right)
\end{aligned}
$$

Now, when measuring the coincidences, the detectors see a combination of the counts from both the primary modes and the mismatch modes (see Fig.3). For example, when detecting coincidences of horizontally polarized photons, the count rate becomes

$$
\begin{aligned}
\left\langle n_{c H_{D}} n_{t H_{D}}\right\rangle & =\left\langle n_{c H_{O}}\left(n_{t H_{O}}+n_{t H_{m}}\right)\right\rangle \\
& =\left\langle n_{c H_{O}} n_{t H_{O}}\right\rangle+\left\langle n_{c H_{O}} n_{t H_{m}}\right\rangle \text { and similarly, } \\
\left\langle n_{c H_{D}} n_{t V_{D}}\right\rangle & =\left\langle n_{c H_{O}} n_{t V_{O}}\right\rangle+\left\langle n_{c H_{O}} n_{t H_{m}}\right\rangle \\
\left\langle n_{c V_{D}} n_{t H_{D}}\right\rangle & =\left\langle n_{c V_{O}} n_{t H_{O}}\right\rangle+\left\langle n_{c V_{O}} n_{t H_{m}}\right\rangle+\left\langle n_{c V_{m}} n_{t H_{O}}\right\rangle+\left\langle n_{c V_{m}} n_{t H_{m}}\right\rangle \\
\left\langle n_{c V_{D}} n_{t V_{D}}\right\rangle & =\left\langle n_{c V_{O}} n_{t V_{O}}\right\rangle+\left\langle n_{c V_{O}} n_{t V_{m}}\right\rangle+\left\langle n_{c V_{m}} n_{t V_{O}}\right\rangle+\left\langle n_{c V_{m}} n_{t V_{m}}\right\rangle
\end{aligned}
$$

These moments are summarized for logical inputs in Table 3. As expected, the mode mismatch has not affected the CNOT operation when the control is "off" (i.e. when $c_{H}$ is occupied). In this case, there is no interaction at beamsplitter $B_{2}$ (Fig.3) and thus no non-classical interference. However, when the control is "on", the effects of the mismatch are noticeable.

\begin{tabular}{|c|c|c|c|c|}
\hline Input & $\left\langle n_{c H_{D}} n_{t H_{D}}\right\rangle$ & $\left\langle n_{c H_{D}} n_{t V_{D}}\right\rangle$ & $\left\langle n_{c V_{D}} n_{t H_{D}}\right\rangle$ & $\left\langle n_{c V_{D}} n_{t V_{D}}\right\rangle$ \\
\hline \hline$|H\rangle_{c}|H\rangle_{t}$ & $\frac{1}{9}$ & 0 & 0 & 0 \\
\hline$|H\rangle_{c}|V\rangle_{t}$ & 0 & $\frac{1}{9}$ & 0 & 0 \\
\hline$|V\rangle_{c}|H\rangle_{t}$ & 0 & 0 & $\frac{2}{9}(1-\xi)$ & $\frac{1}{9}$ \\
\hline$|V\rangle_{c}|V\rangle_{t}$ & 0 & 0 & $\frac{1}{9}$ & $\frac{2}{9}(1-\xi)$ \\
\hline
\end{tabular}

Table 3. Coincident expectation values calculated for each of the four logical basis inputs, with mode matching $\xi$.

Interestingly, the mismatch adds extra terms rather than redistributing the probabilities of the counts measured in the ideal case. Coincidence events which previously were disallowed due to the non-classical interference can now appear as error events because of the mismatch. Thus the probabilities that are being redistributed are those for the states that were not detected in the ideal case (the states which had been postselected out).

We now consider the performance of the gate as a Bell state analyzer in the presence of mode mismatch. As in the ideal case, another beamsplitter is added to the outputs of the control qubit. Another ancillary mode $v_{4}$ must be added to interact with the mismatch mode $c_{V_{m}}$.

The beamsplitter outputs are given in the Heisenberg picture by

$$
\begin{aligned}
c_{S_{1 O}} & =\frac{1}{\sqrt{2}}\left(c_{H_{O}}+c_{V_{O}}\right) \\
c_{S_{1 M}} & =\frac{1}{\sqrt{2}}\left(v_{4}+c_{V_{m}}\right) \\
c_{S_{2 O}} & =\frac{1}{\sqrt{2}}\left(c_{H_{O}}-c_{V_{O}}\right) \\
c_{S_{2 M}} & =\frac{1}{\sqrt{2}}\left(v_{4}-c_{V_{m}}\right)
\end{aligned}
$$

Each detector receives counts from both of the modes incident on it, so the expectation values must be combined in a similar way to Eq. 10. The coincidence count rates are given in Table 4 . Using $\xi=1$ yields the perfectly matched case calculated previously (see Table 2). The error probability for Bell state discrimination is plotted in Fig.4. For small mismatch the error is approximately equal to the percentage mismatch. Clearly good Bell state discrimination will require accurate mode matching to the central beamsplitter. 
Error probability

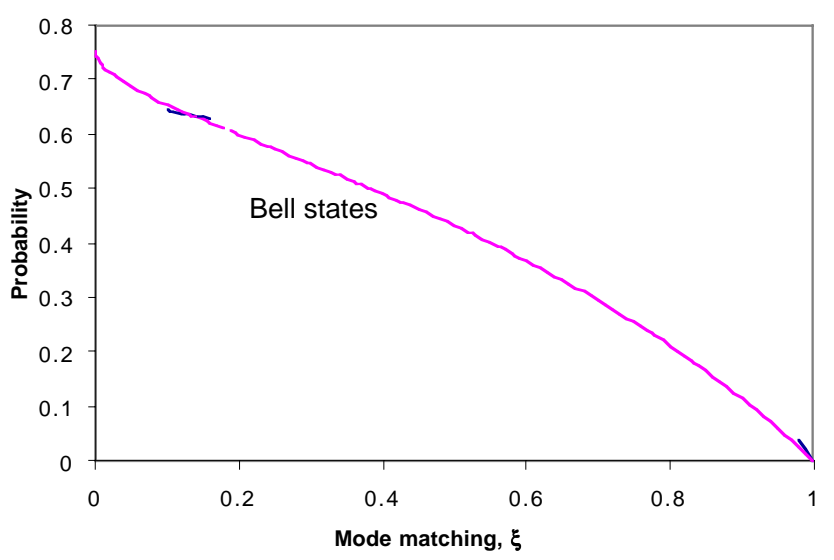

Figure 4. Relative error rates as a function of mode matching for the four Bell states.

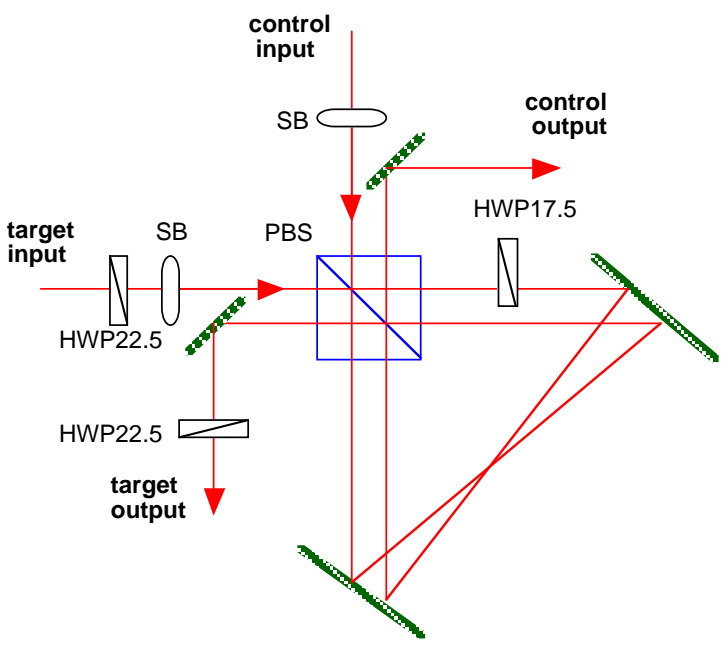

Figure 5. Schematic of experimental CNOT optical circuit. The displaced Sagnac configuration gives interferometric stability without active locking. The various components are PBS: polarizing beamsplitter; $H P W \phi$ : half-wave plate oriented at $\phi$ and; SB: state biasing element. The state biasing elements balance the classical interference in the circuit 


\begin{tabular}{|c|c|c|c|c|}
\hline Input & $\left\langle n_{c S_{1}} n_{t H_{D}}\right\rangle$ & $\left\langle n_{c S_{2}} n_{t H_{D}}\right\rangle$ & $\left\langle n_{c S_{1}} n_{t V_{D}}\right\rangle$ & $\left\langle n_{c S_{2}} n_{t V_{D}}\right\rangle$ \\
\hline \hline$\left|\psi^{+}\right\rangle$ & $\frac{1}{18}(1+\sqrt{\xi})$ & $\frac{1}{18}(1-\sqrt{\xi})$ & $\frac{1}{18}(1-\xi)$ & $\frac{1}{18}(1-\xi)$ \\
\hline$\left|\psi^{-}\right\rangle$ & $\frac{1}{18}(1-\sqrt{\xi})$ & $\frac{1}{18}(1+\sqrt{\xi})$ & $\frac{1}{18}(1-\xi)$ & $\frac{1}{18}(1-\xi)$ \\
\hline$\left|\phi^{+}\right\rangle$ & $\frac{1}{18}(1-\xi)$ & $\frac{1}{18}(1-\xi)$ & $\frac{1}{18}(1+\sqrt{\xi})$ & $\frac{1}{18}(1-\sqrt{\xi})$ \\
\hline$\left|\phi^{-}\right\rangle$ & $\frac{1}{18}(1-\xi)$ & $\frac{1}{18}(1-\xi)$ & $\frac{1}{18}(1-\sqrt{\xi})$ & $\frac{1}{18}(1+\sqrt{\xi})$ \\
\hline
\end{tabular}

Table 4. Coincident expectation values calculated in the superposition basis for the four Bell states when there is mode mismatch present.

\section{EXPERIMENTAL ARRANGEMENT}

The gate arrangement depicted in Fig.1 is composed of two classical interferometers coupled non-classically, representing quite an experimental challenge. To avoid the requirement of active stabilization we have opted for a displaced Sagnac arrangement as shown in Fig.5. By making a displaced polarization Sagnac both classical interferometers are superimposed in the single interferometer. The non-classical coupling between them is achieved via a wave-plate inserted into only one arm. By orienting this beamsplitter at 17.5 degrees the mixing is equivalent to a $1 / 3$ beamsplitter. The $50: 50$ beamsplitters on the target mode are similarly implemented via half-wave plates oriented at 22.5 degrees on the polarization encoded target in and target out beams. The losses needed to balance the classical interferometers (beamsplitters $B 1$ and $B 5$ in Fig.1) can be introduced by state biasing elements outside the interferometer. Strictly these should be polarization dependent loss elements, but for demonstration purposes simple polarization rotations will suffice.

We have been able to achieve high stability and good mode matching with this arrangement and expect to demonstrate CNOT operation in the near future.

\section{CONCLUSION}

Before KLM it was thought that any non-trivial two qubit gate in optics would require very strong Kerr nonlinearity. Now it is known that non-trivial coincidence basis gates can be built from relatively simple linear networks and that, in principle, these gates can made scalable by the inclusion of additional ancilla photons (and considerable increase in circuit complexity and technical requirements). Our aim is to demonstrate such a gate with high fidelity and stability which will not only demonstrate the KLM principles but also may find applications in quantum communications and small scale demonstrations of quantum optical circuits. ${ }^{12,13}$

\section{ACKNOWLEDGMENTS}

This work was supported by the Australian Research Council and ARDA.

\section{REFERENCES}

1. P. G. Kwiat, K. Mattle, H. Weinfurter, A. Zeilinger, A. V. Sergienko, and Y. Shih Phys.Rev.Lett 75, 4337 (1995).

2. J. Brendel, N. Gisin, W. Tittel, Phys.Rev.Lett 82, 2594 (1999).

3. A. G. White, D. F. V. James, P. H. Eberhard and P. G. Kwiat, Phys.Rev.Lett 83, 3103 (1999).

4. G. J. Milburn, Phys.Rev.Lett 62, 2124 (1988).

5. E. Knill, R. Laflamme and G. Milburn, Nature 409, 46, (2001).

6. K. Sanaka, K. Kawahara and T. Kuga, Phys.Rev.A 66, 040301(R) (2002)

7. W. Dür and H.-J. Briegel Phys. Rev. Lett. 90, 067901 (2003).

8. T. B. Pittman, M. J. Fitch, B. C. Jacobs and J. D. Franson, quant-ph/0303113 (2003).

9. T. C. Ralph, N. K. Langford, T. B. Bell and A. G. White, Phys.Rev.A 65, 062324 (2002).

10. H. F. Hofmann, S. Takeuchi, quant-ph/0111092 (2001).

11. In general the Schrödinger evolution for a linear network is obtained by inverting the Heisenberg equations (i.e. expressing the inputs as functions of the outputs). 
12. T. C. Ralph, to appear in IEEE Journal of Selected Topics in Quantum Electronics; quant-ph/0306190 (2003).

13. J. L. Dodd, T. C. Ralph, G. J. Milburn, quant-ph/0306081 (2003). 